\title{
Cordycepin activates autophagy through AMPK phosphorylation to reduce abnormalities in Machado-Joseph disease models
}

\author{
Adriana Marcelo ${ }^{1,2,3,4, \neq}$, Filipa Brito ${ }^{4, \neq}$, Sara Carmo-Silva ${ }^{4}$, Carlos A. Matos ${ }^{4,5}$, \\ João Alves-Cruzeiro ${ }^{4}$, Ana Vasconcelos-Ferreira ${ }^{4}$, Rebekah Koppenol ${ }^{1}$, \\ Liliana Mendonça ${ }^{4}$, Luís Pereira de Almeida ${ }^{4,6, \neq}$ and Clévio Nóbrega ${ }^{1,2,3,4, \uparrow, \neq, *}$
}

${ }^{1}$ Centre for Biomedical Research (CBMR), University of Algarve, Portugal, ${ }^{2}$ Department of Biomedical Sciences and Medicine (DCBM), University of Algarve, Portugal, ${ }^{3}$ Algarve Biomedical Center (ABC), University of Algarve and University Hospital of Algarve, Portugal, ${ }^{4}$ Center for Neuroscience and Cell Biology (CNC), University of Coimbra, Portugal, ${ }^{5}$ Institute for Interdisciplinary Research, University of Coimbra, Portugal and ${ }^{6}$ Faculty of Pharmacy, University of Coimbra, Portugal

*To whom correspondence should be addressed at: Clévio Nóbrega Department of Biomedical Sciences and Medicine University of Algarve Campus de Gambelas 8005-139 Faro, Portugal. Tel: +35191797250; Email: cdnobrega@ualg.pt

\begin{abstract}
Machado-Joseph disease (MJD) is a neurodegenerative disorder caused by an abnormal expansion of citosine-adenine-guanine trinucleotide repeats in the disease-causing gene. This mutation leads to an abnormal polyglutamine tract in the protein ataxin-3 (Atx3), resulting in formation of mutant Atx3 aggregates. Despite several attempts to develop a therapeutic option for MJD, currently there are no available therapies capable of delaying or stopping disease progression. Recently, our group reported that reducing the expression levels of mutant Atx3 lead to a mitigation of several MJD-related behavior and neuropathological abnormalities. Aiming a more rapid translation to the human clinics, in this study we investigate a pharmacological inhibitor of translation-cordycepin—in several preclinical models. We found that cordycepin treatment significantly reduced (i) the levels of mutant Atx3, (ii) the neuropathological abnormalities in a lentiviral mouse model, (iii) the motor and neuropathological deficits in a transgenic mouse model and (iv) the number of ubiquitin aggregates in a human neural model. We hypothesize that the effect of cordycepin is mediated by the increase of phosphorylated adenosine monophosphate-activated protein kinase (AMPK) levels, which is accompanied by a reduction in the global translation levels and by a significant activation of the autophagy pathway. Overall, this study suggests that cordycepin might constitute an effective and safe therapeutic approach for MJD, and probably for the other polyglutamine diseases.
\end{abstract}

\footnotetext{
†Clévio Nóbrega, http://orcid.org/0000-0002-8312-5292

† These authors contributed equally to this work.

Received: June 24, 2018. Revised: September 10, 2018. Accepted: September 11, 2018

(c) The Author(s) 2018. Published by Oxford University Press. All rights reserved.

For Permissions, please email: journals.permissions@oup.com
} 


\section{Introduction}

Machado-Joseph disease (MJD), or spinocerebellar ataxia type 3, is an autosomal dominantly inherited neurodegenerative disorder. It belongs to the polyglutamine (polyQ) diseases group, which is caused by consecutive repetitions of the trinucleotide citosine-adenine-guanine in the coding region of the diseasecausing genes, translating to a pathogenic polyQ tract in the corresponding proteins (7). Regarding MJD, the causative gene is MJD1 and the mutated protein is ataxin-3 (Atx3), a deubiquitinating enzyme which is thought to play a role in the ubiquitinmediated proteolysis (9). In a normal situation, Atx3 contains 10-51 glutamines, while when mutated this protein carries 5584 glutamines (12). The polyQ tract in Atx3 induces protein aggregation and accumulation of insoluble ubiquitin-positive aggregates of mutant Atx3 inside neuronal cells, leading to progressive neurodegeneration in several brain regions, including the cerebellum, substantia nigra and spinal cord (28). This severe neuropathological phenotype results in numerous symptoms, such as progressive ataxia, dysphagia, dysarthria and rigidity among others (32). Currently, there is no effective treatment to delay or stop disease progression.

In the past years, several therapeutic approaches were studied in different preclinical models, such as RNA interferencebased strategies $(1,20)$, the activation of protein clearance systems (15) or stem cells transplantation (13). However, none of these strategies were yet translated to human application. Therefore, there is an urgent need to develop therapeutic strategies for MJD and for the other polyQ diseases, which could be easily translated to the clinics.

Cordycepin or $3^{\prime}$-deoxyadenosine is a bioactive compound found in the fungi Cordyceps and a derivative of the nucleoside adenosine (22). It was suggested that cordycepin might activate the adenosine monophosphate-activated protein kinase (AMPK) pathway, thus reducing protein translation levels (31). Recently, we reported that ataxin-2 overexpression reduced the expres- sion levels of mutant Atx3, through the reduction of the Atx3 translation (17). Thus, we suggested that targeting mutant Atx3 translation could be a potential therapeutic strategy for MJD, as it resulted in alleviation of the severe phenotype observed in different preclinical models. Nevertheless, the modulation of translation levels was achieved by a lentiviral-based therapy, which is still far away to be used in a clinical context. Thus, in this study we aimed at investigating a pharmacological treatment based in the administration of cordycepin in several MJD preclinical models, as well as to characterize its molecular mechanism of action. We observed that cordycepin administration decreased the levels of mutant Atx3 aggregates in in vitro and in vivo models, resulting in the amelioration of the associated abnormalities of lentiviral and transgenic mouse models. Furthermore, we reported that this cordycepin effect is mediated by activation of AMPK pathway, independently of the mammalian target of rapamycin (mTOR) pathway, resulting in the reduction of the global translation levels and in a significant activation of the autophagy pathway. Overall, this study suggested for the first time that cordycepin might constitute a therapeutic approach for MJD, and probably to other polyQ diseases.

\section{Results}

\section{Cordycepin treatment reduces the mutant Atx3 levels in N2A cells}

Previously, we reported that the molecular regulation of mutant Atx3 translation resulted in alleviation of the MJD phenotype (17). In line with these results, we found that cordycepin (translation inhibitor) treatment decreased mutant Atx3 (72Q) levels in a mouse neuroblastoma cell line (N2A cells) model (Fig. 1A and C), whereas no alterations were detected in mouse endogenous Atx3 (Fig. 1A-B, 1D) or in human wild-type Atx3 (27Q) levels (Fig. 1B and E).
A

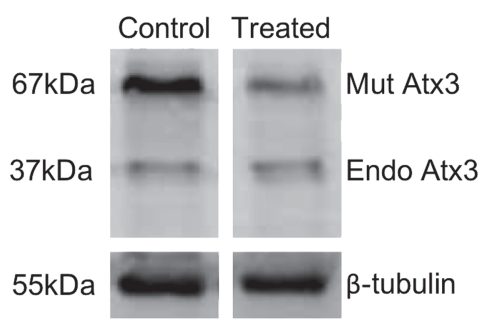

C
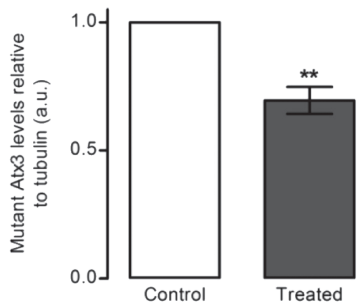

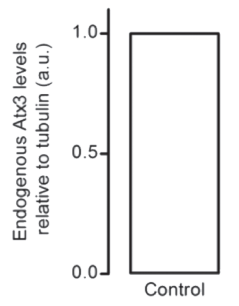

B

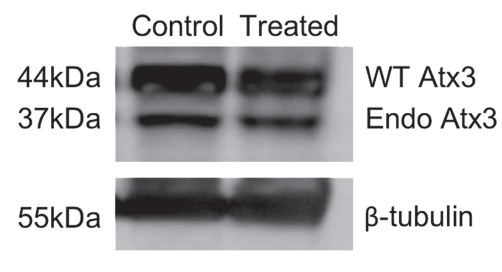

E
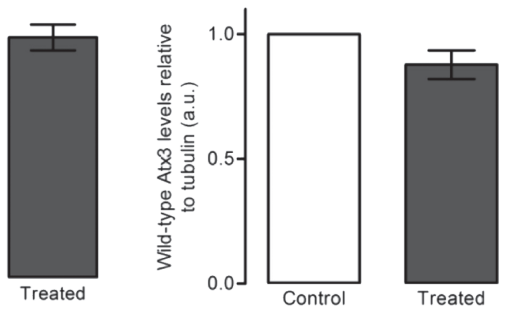

Figure 1. Cordycepin decreases the levels of mutant Atx3 in N2A cells. Western blot of protein lysates from N2A cells expressing human full-length mutant Atx3Q72 (A) and human wild-type Atx3 with 27 glutamines (B) treated with $20 \mu \mathrm{M}$ cordycepin for $48 \mathrm{~h}$ and non-treated (control) ( $n=4$ independent experiments). The cordycepin treatment decreased the levels of mutant Atx3 compared to control levels (C), whereas mouse endogenous Atx3 remained unaltered (D) as well as wild-type Atx3 protein (E). Results were expressed as mutant, endogenous or wild-type Atx3 levels over tubulin ratio. Values are expressed as mean \pm SEM. ${ }^{* *} P<0.01$ (unpaired Student's t-test). 
A

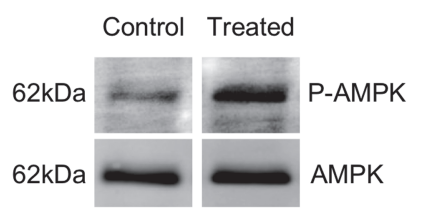

C

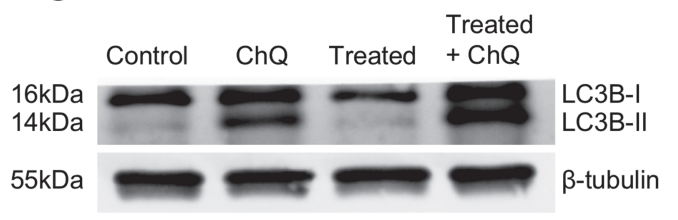

E

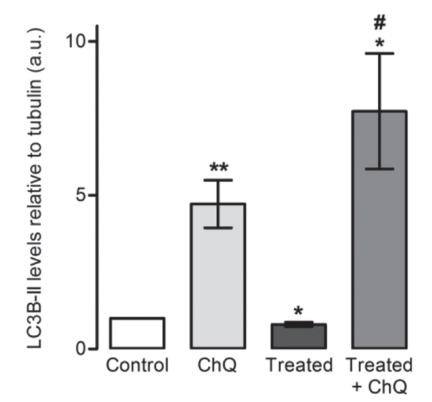

B

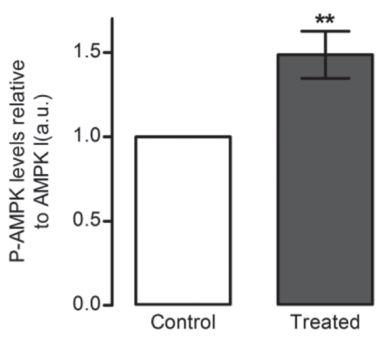

D

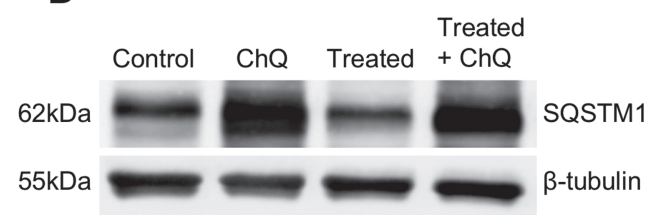

G

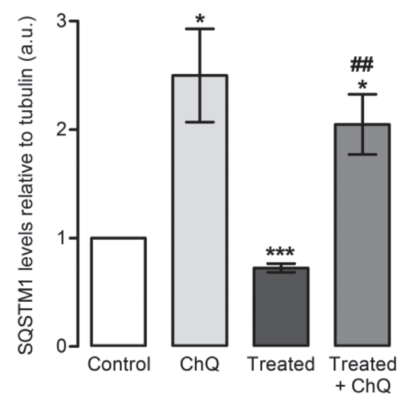

I

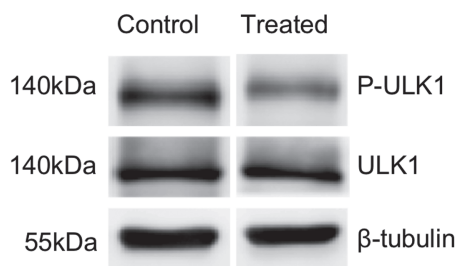

K

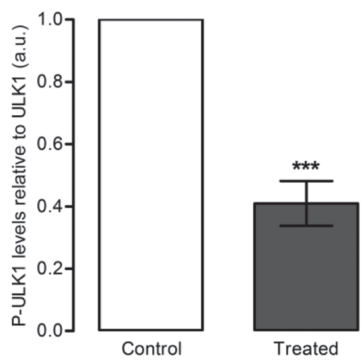

Figure 2. Cordycepin induces AMPK activation promoting autophagy in N2A cells. (A) Western blot of protein lysates from N2A cells treated with 20 MM cordycepin for $48 \mathrm{~h}$ and non-treated (control) ( $n=6$ independent experiments). (B) The treatment with cordycepin led to a significant increase in the levels of P-AMPK relative to total AMPK levels when compared to control, suggesting an activation of AMPK signaling pathway. Western blot analysis of LC3B-II (C) and SQSTM1 (D) levels ( $n=3$ independent experiments). Cordycepin treatment decreased the levels of LC3B-II (E) and SQSTM1 (G), while an accumulation of these subtracts was detected in the presence of ChQ. (F) LC3B-II net flux was calculated by subtracting the amount of LC3B-II in treated condition from the condition treated with ChQ. Cordycepin induced an increase in LC3B-II net flux compared to control levels, suggesting an activation of the autophagic flux. Western blot analysis of P-mTOR and mTOR (H) and P-ULK1Ser555 and ULK1 (I) levels ( $n=4$ independent experiments). Cordycepin treatment resulted in a significant increase of the P-mTOR relative to total mTOR levels (J) and in a significant decrease in P-ULK1 relative to total ULK1 levels (K). Values are expressed as mean $\pm \mathrm{SEM}$. ${ }^{*} \mathrm{P}<0.05,{ }^{* *} \mathrm{P}<0.01 ;{ }^{* * *} \mathrm{P}<0.001$ (one-way ANOVA relative to control levels). ${ }^{\#} \mathrm{P}<0.05,{ }^{\# \# P}<0.01$ (unpaired Student's t-test of treated and treated + ChQ levels). 


\section{Cordycepin activates AMPK, increasing its phosphorylation, inducing autophagy and reducing overall translation levels in N2A cells}

Next, we tried to elucidate the molecular mechanism behind the cordycepin effect in reducing mutant Atx3 levels. Previously, it was hypothesized that cordycepin inhibits translation through activation of AMPK pathway (31). In line with this, we found that cordycepin significantly increased the levels of phosphorylated AMPK (P-AMPK) in N2A cells expressing human full-length mutant Atx3 (Fig. 2A and B). This increase was accompanied by a significant decrease in the global protein synthesis levels (Supplementary Material, Fig. 1). These results raised the hypothesis that cordycepin might also impact autophagy induced by AMPK activation (11). In line with this, we next assessed the levels of two autophagic markers, LC3B-II (Fig. 2C) and sequestosome-1 (SQSTM1; Fig. 2D). After cordycepin treatment, we observed a decrease in both LC3B-II (Fig. 2C and E) and
SQSTM1 protein levels (Fig. 2D and G), suggesting an increase of autophagic clearance. To confirm that cordycepin was, in fact, enhancing autophagy, we evaluated LC3B-II and SQSTM1 levels in the absence and presence of chloroquine (ChQ), an inhibitor of autolysosomal degradation. In the presence of ChQ both LC3B-II and SQSTM1 accumulate upon cordycepin treatment (Fig. $2 \mathrm{C}$ and D). In addition, when comparing the differences in the amount of LC3B-II between samples in the presence and absence of ChQ (autophagic flux), we observed that cordycepin significantly increased autophagic flux in N2A cells, expressing human full-length mutant Atx3 (Fig. 2F). Altogether, these results demonstrate that cordycepin activates autophagy. To further investigate the impact of cordycepin in autophagy activation, we next studied the MTOR, a negative regulator of autophagy (3) and UNC-51-like autophagy activating kinase 1 (ULK1), an autophagy activating enzyme levels. We found that cordycepin treatment significantly increased the levels of phosphorylated mTOR (P-mTOR) (Fig. $2 \mathrm{H}$ and J), whereas a
A

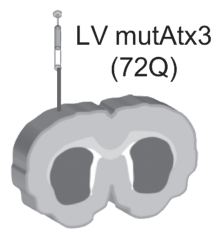

C

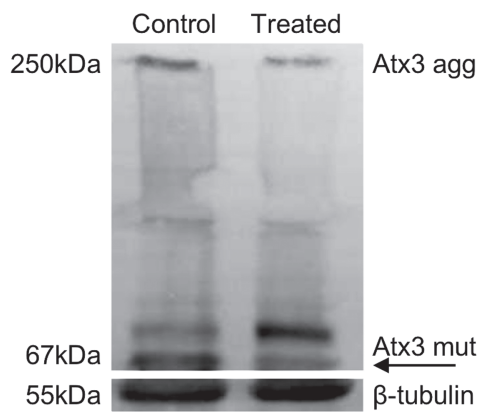

$\mathrm{F}$

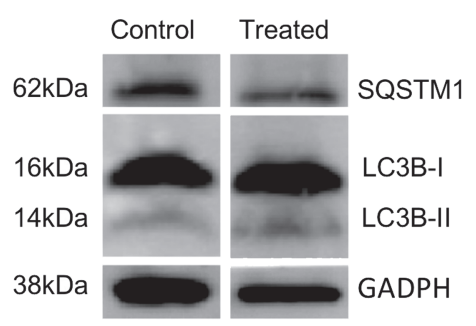

B

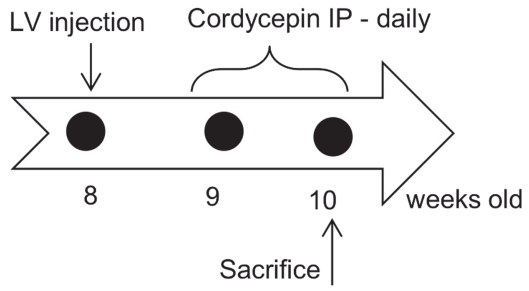

$\mathrm{E}$
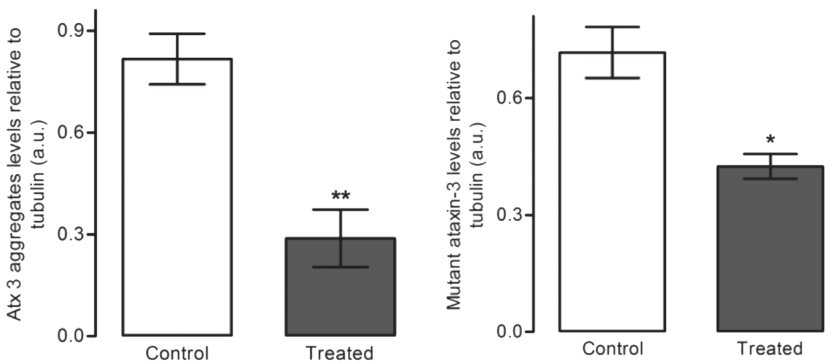

$\mathrm{H}$

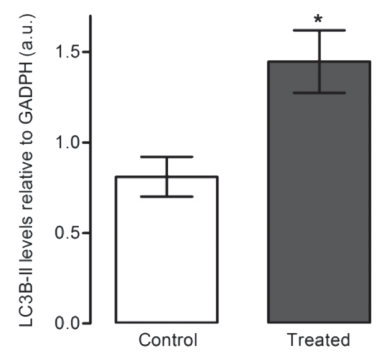

Figure 3. Cordycepin reduces mutant Atx3 levels in a lentiviral MJD mouse model through the activation of autophagy. (A) Schematic representation of the stereotaxic injection of LV encoding for mutant Atx3Q72 in the right hemisphere of the striatum (B). Timeline of the experiment; the animals received the LV injection at 8 weeks old and cordycepin treatment started one week later. Treated animals $(n=3)$ were administered with a daily intraperitoneal injection of $20 \mathrm{mg} / \mathrm{Kg}$ of cordycepin$\mathrm{NaCl}$ 0.1\%, whereas control group $(n=3)$ received the vehicle $(\mathrm{NaCl}$ 0.1\%) during 1 week. Western blot analysis of Atx3 protein (C) and LC3B-II and SQSTM1 proteins (F) of striatal punches of animals' brain. Cordycepin treatment significantly decreases the levels of Atx3 aggregates (D) and soluble mutant Atx3 (E). Cordycepin administration resulted in a significant increase in LC3B-II autophagic marker (G) as well as a reduction of SQSTM1 autophagic protein (H), thus suggesting an activation of autophagy. Results were expressed as mutant Atx3 aggregates or soluble protein over tubulin levels and as LC3B-II or SQSTM1 over GADPH levels. Values are expressed as mean \pm SEM. ${ }^{*} P<0.05,{ }^{* *} P<0.01$ (unpaired Student's t-test). 
A

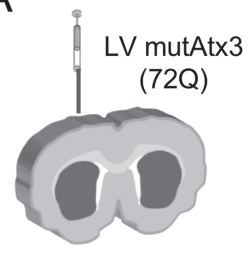

Control
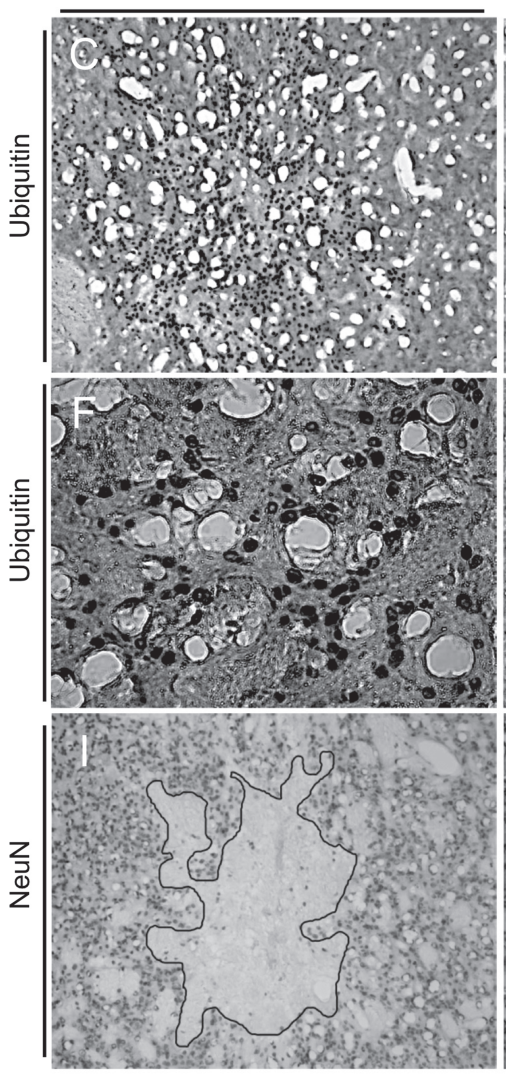

$\mathrm{L}$

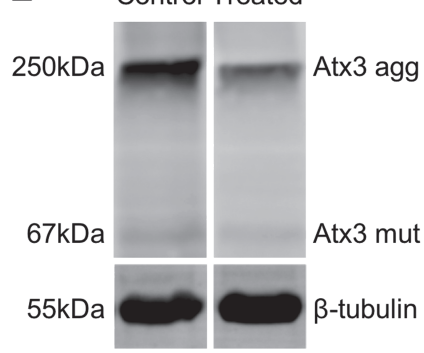

B

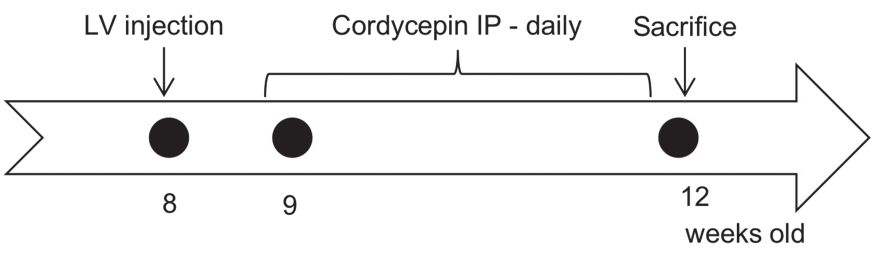

Treated

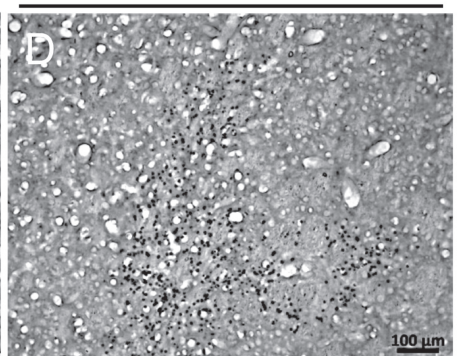

E

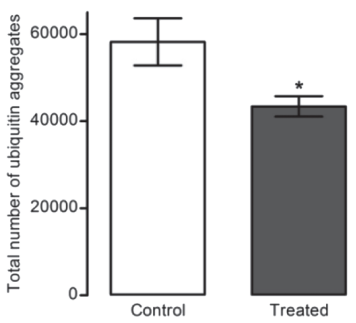

$\mathrm{H}$

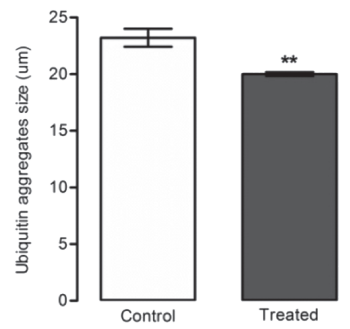

$\mathrm{K}$

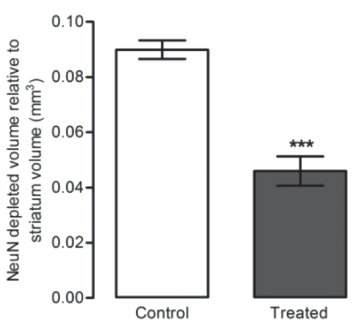

$\mathrm{N}$

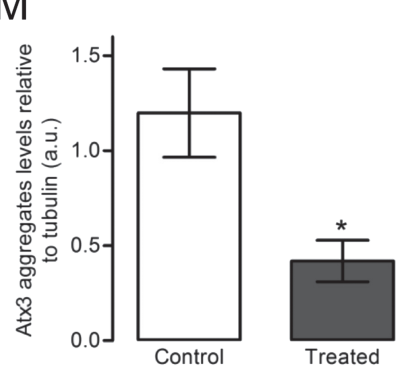

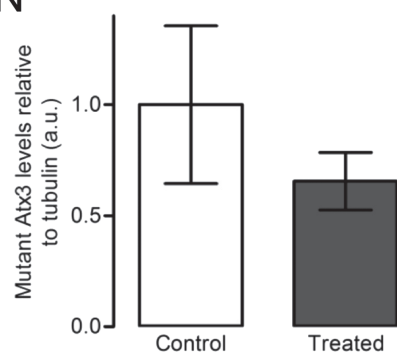

Figure 4. Cordycepin mitigates neuropathological deficits and reduces mutant Atx3 levels in a lentiviral MJD mouse model. (A) Schematic representation of a stereotaxic injection of LV encoding for mutant Atx3Q72 in the right hemisphere of the striatum (B). Timeline of the experiment; the animals received the LV injection at 8 weeks old and cordycepin treatment started one week later. Treated animals $(n=9)$ were administered a daily intraperitoneal injection of $20 \mathrm{mg} / \mathrm{Kg}$ of cordycepin-NaCl $0.1 \%$, whereas control group $(n=9)$ received the vehicle ( $\mathrm{NaCl} 0.1 \%$ ). Treatment was prolonged for 4 weeks. (C, D, F, G) Immunohistochemistry staining for ubiquitin depicting mutant Atx3 aggregates. Animals treated with cordycepin $(n=4)$ showed a significant decrease in the number $(\mathrm{E})$ as well as the size $(\mathrm{H})$ of the inclusions compared to control group $(n=4)$. (I, J) Immunohistochemistry staining for the neuronal marker NeuN. (K) Cordycepin treatment $(n=4)$ resulted in a preservation of NeuN staining volume compared to non-treated condition $(n=4)$. Results were expressed as NeuN-depleted volume relative to total striatum volume. (L) Western blot of striatal punches of the lentiviral model ( $n=5$ for both groups). The cordycepin treatment reduced the levels of mutant Atx3 aggregates (M) and mutant soluble Atx3 protein (N). Results were expressed as mutant Atx3 aggregates or soluble protein over tubulin levels. Values are expressed as mean \pm SEM. ${ }^{*} P<0.05$, ${ }^{* *} P<0.01,{ }^{* * *} P<0.001$ (unpaired Student's t-test). 

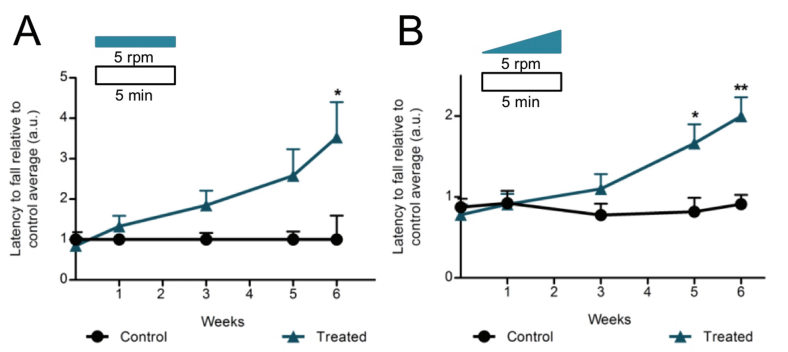

C
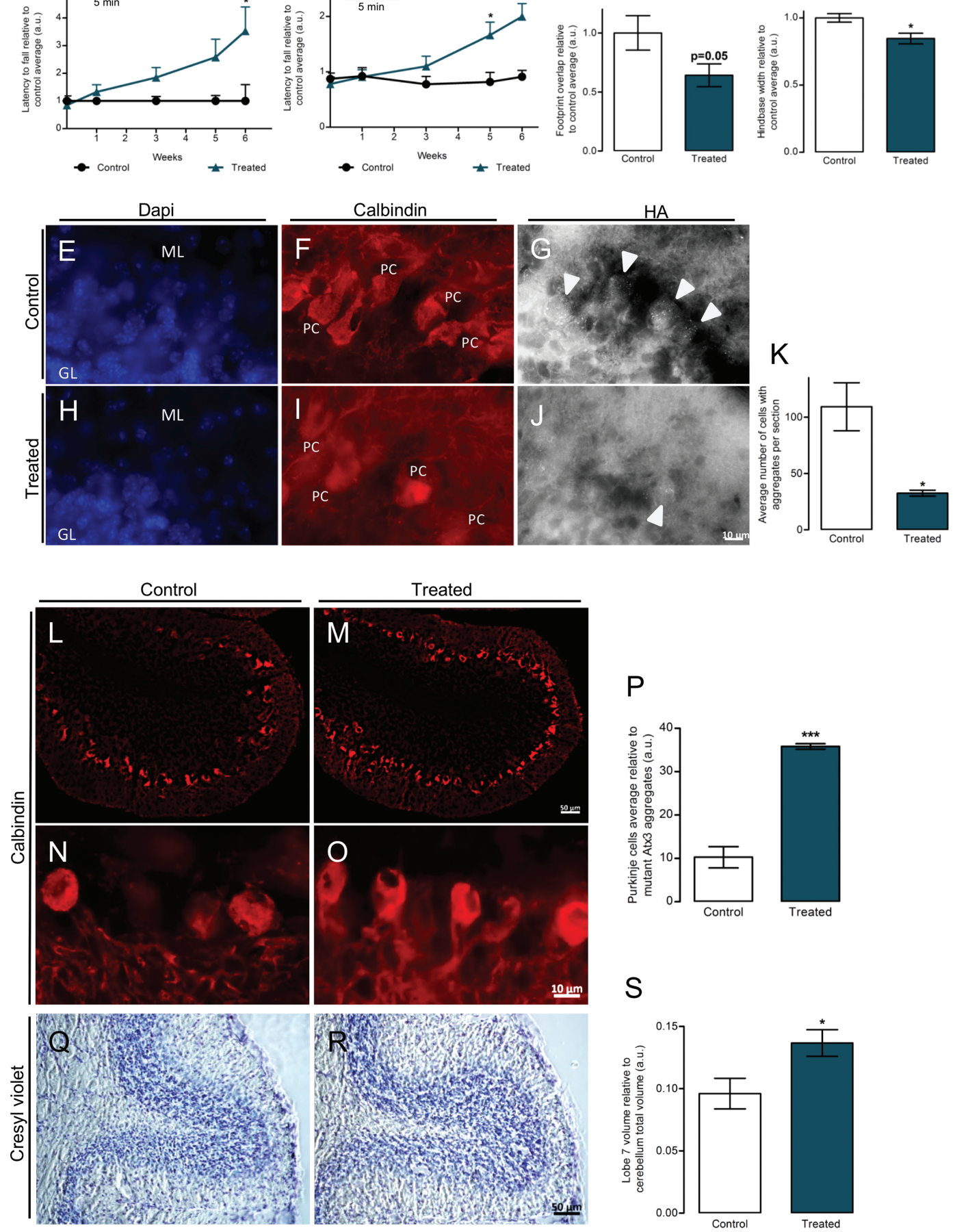

Figure 5. Cordycepin ameliorates motor performance and neuropathology in a transgenic MJD mouse model. (A-D) Behavioral assessment of the animals started before the treatment ( 0 weeks) and it was performed every 2 weeks until the end of the experiment ( 6 weeks). Animal motor coordination and balance were evaluated through constant (A) and accelerated rotarod tests (B). Mice treated with cordycepin $(n=10)$ stayed for long in the rotating rod at 6 weeks (A) post-injection for constant speed and at 5 and 6 weeks for increased velocity (B), compared to non-treated animals $(n=8)$. The results were normalized to the control average for each time point. Values are presented as mean \pm SEM. ${ }^{*} P<0.05,{ }^{* *} P<0.01$ (two-way ANOVA). Cordycepin treatment $(n=10)$ showed a decrease in footprint overlap (C) and a significant decrease in hind base width measures (D) compared to control condition $(n=8)$. (E-J) Representative fluorescence microscopy images detailing DAPI, calbindin and HA staining's for cordycepin treated animals (H-J) and control group (E-G). Arrowheads point towards cells with HA aggregates. Administration of cordycepin led to a robust decrease in the number of cells with mutant Atx3 aggregates in the cerebellum (K). (L-O) Representative fluorescence microscopy images of calbindin staining for mice treated with cordycepin ( $\mathrm{M}, \mathrm{O})$ and non-treated group $(\mathrm{L}, \mathrm{N})$. Results showed a significant increase of Purkinje cells in the treated group compared to control animals (P). Representative microscopy images of cresyl violet histological staining for treated group (R) and non-treated mice (Q). Cordycepin treatment resulted in a significant decrease in cerebellar lobe 7 volume compared to non-treated animals (S). Results are expressed as lobe 7 volume relative to cerebellum total volume. Values are expressed as mean \pm SEM. ${ }^{*} P<0.05,{ }^{* * *} P<0.001$ (unpaired Student's t-test). ML, molecular layer; GL, granular layer; PC, Purkinje cells. 
significant reduction in phosphorylated ULK1 at Ser555 (P-ULK1Ser555) levels (Fig. 2I and K) was observed. Altogether, these results suggest that cordycepin leads to a strong activation of autophagy through mTOR-independent pathway.

\section{Cordycepin reduces mutant Atx3 protein levels through activation of autophagy in a lentiviral MJD mouse model}

After the in vitro validation of cordycepin treatment, we next aimed at investigating whether this compound could reduce the levels of mutant Atx3 in a lentiviral mouse model of MJD. For that, animals were injected, in the right hemisphere of the striatum, with lentiviral vectors (LVs) encoding for mutant Atx3 with 72 glutamines (Atx3Q72) (Fig. 3A). Cordycepin intraperitoneal injections (IP) were administered daily for 1 week, starting 1 week post-LV injection (Fig. 3B). Western blot analysis of brain striatal punches (Fig. 3C) showed that cordycepin treatment led to a significant decrease of both mutant Atx3 aggregates (Fig. 3D) and soluble mutant Atx3 (Fig. 3E) levels compared to the control group, treated with saline solution. Moreover, we assessed the levels of LC3B-II and SQSTM1 (Fig. 3F) and observed that cordycepin treatment significantly increases LC3B-II levels (Fig. 3G) and reduced SQSTM1 levels (Fig. 3H), which is line with studies using pharmacological agents to induce autophagy in vivo and thus suggesting that cordycepin might be activating autophagy in vivo (14). These results indicate that cordycepin can effectively reduce mutant Atx3 levels in an in vivo MJD model through activation of the autophagic pathway.
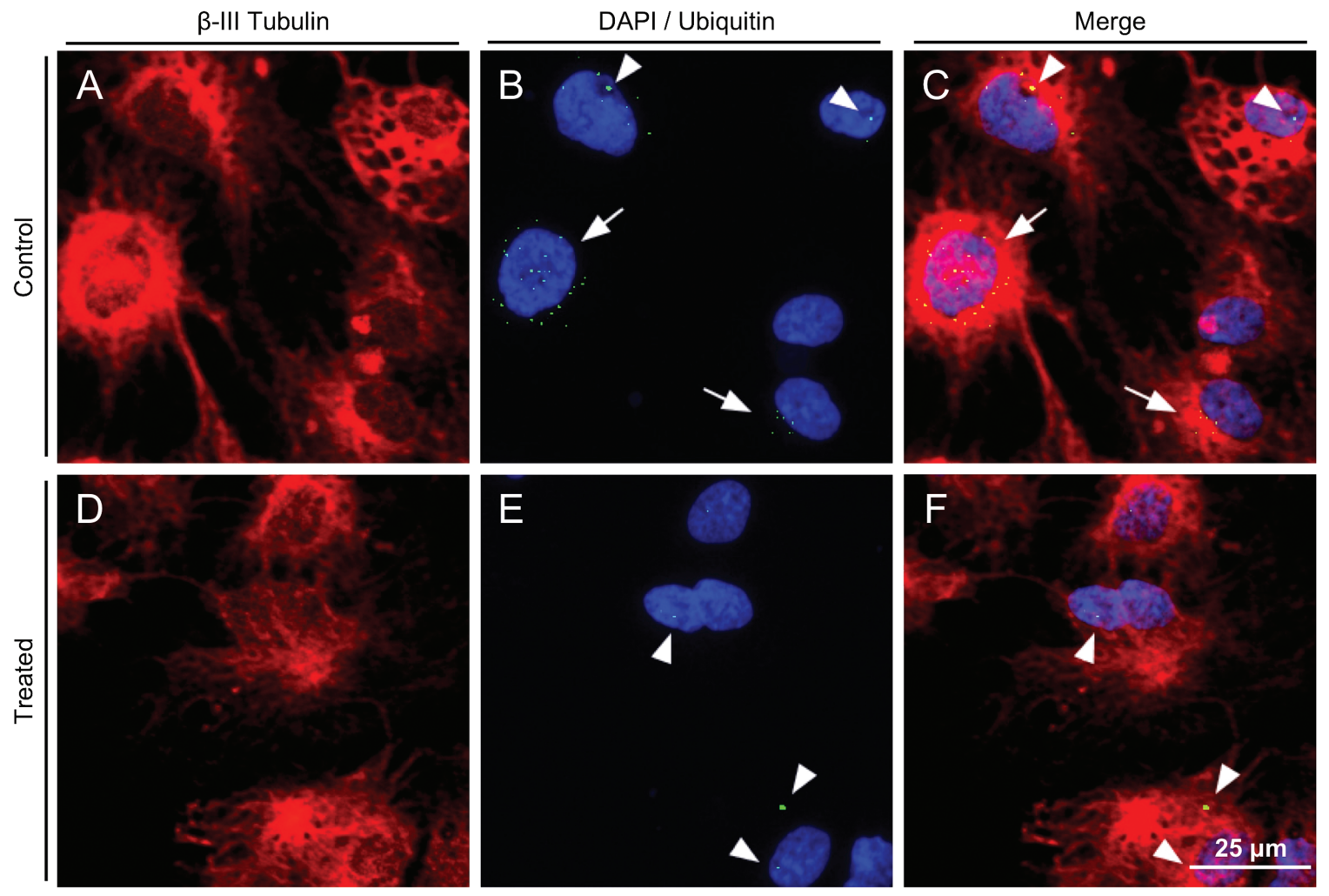

G

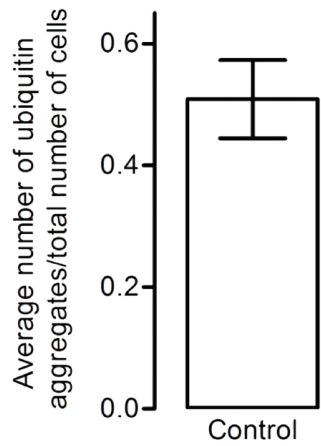

$\mathrm{H}$

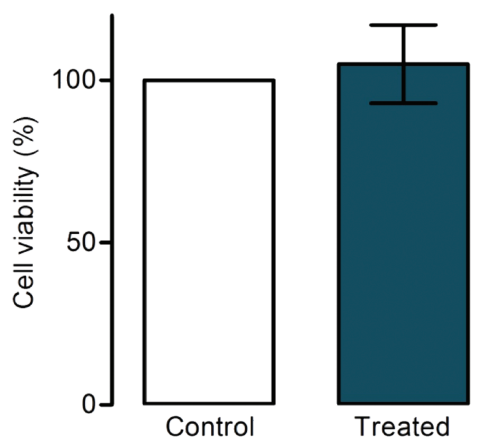

Figure 6. Cordycepin decreases the number of ubiquitin aggregates in a human neural MJD model. Immunocytochemistry staining for ubiquitin (B, E) and tubulin proteins (A, D). Arrowheads point toward ubiquitinated inclusions and arrows point to cells containing aggregates. Cordycepin treatment ( $n=4)$ decreased the number of ubiquitin-positive aggregates in treated cells compared to non-treated cells (G). (H) Analysis of cell viability through the resazurin reduction assay. Cordycepin administration did not induce cell death, as levels of viability were similar to control group. Values are expressed as mean \pm SEM. * $\mathrm{P}<0.05$ (unpaired Student's t-test). 


\section{Cordycepin treatment ameliorates mutant Atx3-induced abnormalities in a lentiviral MJD mouse model}

The in vivo expression of mutant Atx3 mediated by LV leads to the formation of ubiquitinated aggregates (which are a hallmark of MJD) and the loss of neuronal markers in the striatum and cerebellum $(2,18)$. We next administered a cordycepin treatment in a MJD striatal lentiviral model (Fig. 4A) for 4 weeks, with daily administered IP injections (Fig. 4B). The cordycepin treatment led a significant decrease in the total number of ubiquitinated aggregates (Fig. 4C-E), in the size of the aggregates (Fig. 4F-H) and to a significant preservation of the neuronal marker NeuN (Fig. 4I and K, Supplementary Material, Fig. 2A-B), compared with the control animals treated with saline solution. Moreover, we also observed that cordycepin treatment reduced the mutant Atx3 protein aggregates and soluble levels, detected by western blot (Fig. 4L-N). To evaluate a possible systemic toxicity effect due to the cordycepin treatment, we analyzed the levels of liver enzymes in the blood of treated and nontreated mice. Along with no alteration in the animals' weight, we observe that long-term cordycepin administration did not induce an increase in the levels of alkaline phosphatase (ALP) (Supplementary Material, Fig. 2C) and alanine aminotransferase (ALT) (Supplementary Material, Fig. 2D). These results suggest a strong and safe neuroprotective effect for cordycepin in vivo, mitigating the neuropathological abnormalities in a lentiviral MJD mouse model.

\section{Cordycepin treatment improves motor deficits and neuropathological abnormalities in a MJD transgenic mouse model}

The cerebellum is one of the most affected areas in MJD (27). Thus, we next investigated the effect of cordycepin treatment in a MJD transgenic mouse model with cerebellar pathology (29). As early as 3 weeks of age, this model already displayed cerebellar atrophy, accompanied by imbalance and gait disturbances. We began a 6 weeks treatment with cordycepin (IP) in the animals at 4 weeks of age, where the phenotype and neuropathology are already installed (17). Every 2 weeks, the animals were tested for different behavioral tests (Supplementary Material, Fig. 3). In the rotarod tests, the animals treated with cordycepin showed an increase in the latency to fall times compared with control animals, both at constant rod velocity (Fig. 5A) and with an increasing rod velocity (Fig. 5B). Moreover, the analysis of the footprint patterns at 6 weeks of treatment revealed that cordycepin-treated animals displayed smaller footprint overlap measures (Fig. 5C), as well as a reduction in the hind base measures (Fig. 5D), compared to control animals. We also checked for weight gain during the course of treatment, observing that there were no alterations in body weight upon cordycepin administration (Supplementary Material, Fig. 4). Overall, these results show that cordycepin improves motor coordination and balance, clearly slowing the motor deficits progression, with no apparent toxicity or side effects. We next investigated whether the observed improvements in motor function were correlated with improvements in neuropathological features. This MJD transgenic mouse model expresses a mutant form of Atx3 with a haemagglutinin (HA) epitope in the cerebellar Purkinje cells [(29); Supplementary Material, Fig. 5]. The cordycepin-treated animals showed a significant reduction in the number of cells with HA aggregates, compared to control animals (Fig. 5E-K), as well as a significant increase in the total number of Purkinje cells (Fig. 5L-P). Furthermore, we observed an increased volume in lobes 4, 5, 6 and 8 of the cerebellum in treated animals, being this difference statistically significant in lobe 7 (Fig. 5Q-S). Altogether, these results demonstrate that cordycepin is able to reduce motor and MJD-related neuropathological abnormalities, even after the onset of the disease.

\section{Cordycepin treatment reduces the number of aggregates in an induced pluripotent stem cells MJD model}

To validate the use of cordycepin in a human model, we tested the treatment in a human neuronal model derived from induced pluripotent stem cells (iPSCs) of a MJD patient (4). The cordycepin treatment led to a significant reduction in the number of ubiquitin aggregates, comparing to a control condition (Fig. 6A-G). No differences were detected in the alamar test between both conditions, thus suggesting that cordycepin does not affect cell proliferation/viability (Fig. $6 \mathrm{H}$ ). These results confirm the ability of cordycepin to reduce mutant Atx3 aggregates, with no toxicity for the cells.

\section{Discussion}

MJD is a fatal neurodegenerative disorder that results in serious clinical complications along with a decline of life quality for patients suffering from this condition. In the past years, several studies reported different therapeutic approaches aiming to stop or delay disease progression $(1,8,19,20,26)$. Although these strategies produced encouraging results, currently there are no available therapeutic approaches for MJD patients.

Recently, we showed that molecular modulation through LV of mutant Atx3 translation constitutes a new therapeutic strategy reducing MJD abnormalities (17). Though this strategy can reach high levels of specificity, it is some steps away from a translation to human clinics. Aiming a more rapid and easy translation to the clinics, we investigated a European Medicines Agency- and Food and Drug Administration-approved pharmacologic compound-cordycepin-with several action mechanisms, including translation inhibition in different MJD models. In this study, we found that cordycepin treatment led to a significant and specific decrease in mutant Atx3 levels in an N2A cell model, whereas no alterations were observed in wild-type Atx3 or in endogenous mouse Atx3 levels. The specific effect of cordycepin toward mutant Atx3 while preserving wildtype Atx3 is relevant, as although the Atx3 function is not fully understood its expression throughout the body suggests an important biological role (23). Taking into consideration the promising results obtained in the in vitro experiments, we next investigated the effect of cordycepin treatment in two MJD animal models. In the MJD lentiviral mouse model, animals were injected with LV encoding for mutant Atx3 in the striatum, leading to expression of the mutant Atx3 protein, the formation of ubiquitin-positive mutant Atx3 inclusions and to the loss of neuronal markers (2). Cordycepin treatment led to a reduction in mutant Atx3 aggregates and soluble protein levels detected by western blot, as well as a reduction in the number and size of mutant Atx3 ubiquitinated aggregates, and a significant preservation of the neuronal marker NeuN, comparing to the control animals, thus suggesting a neuroprotective role of cordycepin. Furthermore, cordycepin administration seems to 
not induce toxicity, as confirmed by no alterations in hepatic enzyme levels. The levels of ALT and ALP, two biomarkers for liver damage (10), were not increased in cordycepin-treated animals compared to wild-type mice, which is in agreement with a hepatoprotective effect described for cordycepin (5). We also investigated if cordycepin was able to mitigate disease impairments in a MJD transgenic mouse model with cerebellar pathology (29). Cordycepin treatment ameliorated mice motor coordination and balance, assessed by a significant increase in the latency to fall in the rotarod tests in treated animals, compared with the control animals. Moreover, treated mice also displayed a reduction in the footprint overlap and in the hind base width measurements, indicating improvements in gait impairments. These motor improvements were also accompanied by a significant decrease in the number of intranuclear inclusions, as well as an increase in the number of Purkinje cells, upon cordycepin treatment. These results suggest that cordycepin is able to rescue motor and neuropathological impairments in a setting where the pathology is already established. We also tested cordycepin in a human neural model, differentiated from iPSCs derived from MJD patient fibroblasts (4). Cordycepin administration led to a significant decrease in the number of ubiquitin aggregates compared to the control condition, with no alteration in cell viability. Altogether, these results suggest that cordycepin treatment might constitute an effective and safe therapy for MJD.

Cordycepin was previously shown to act as an important translation inhibitor (31). Thus, we also tried to elucidate the cordycepin molecular action mechanism behind the mitigation of Atx3-induced abnormalities. It was hypothesized that cordycepin could activate AMPK pathway, which enhances the shutdown of the mTOR signaling pathway thus impacting autophagy. We found that cordycepin treatment in our cellular model significantly increased the levels of P-AMPK, which was accompanied by a reduction in the global translation levels. The autophagy pathway is crucial for clearance of misfolded proteins and regulation of protein homeostasis, and it was shown to be deregulated in polyQ diseases, including MJD $(6,16)$. In fact, we already showed that autophagy targeting might constitute a therapeutic option for MJD (15). In this study, our results showed that cordycepin treatment led to an activation of autophagy in vitro and in vivo, assessed by the monitorization of LC3B-II and SQSTM1, two proteins that are degraded in the final stages of the autophagic process. Accordingly, the detected levels of both substrates upon cordycepin treatment suggest an activation of the autophagic pathway, in line with similar studies $(3,14)$. In fact, the autophagic flux measured by the LC3B-II turnover assay showed that upon cordycepin administration there was an increase in the amount of LC3B-II delivered to lysosomes, compared to the control group. Altogether, these results clearly demonstrate that cordycepin enhances autophagy. We further analyzed the P-mTOR levels and P-ULK1 levels (at Ser555), which were reduced upon cordycepin treatment. These results suggest an activation of mTOR pathway and an inhibition of autophagy $(11,30)$, which could indicate that cordycepin is activating autophagy through an independent mTOR pathway.

Overall, in this study, we report a clear and strong neuroprotective effect of cordycepin, mediated by an increase in AMPK phosphorylated levels, an activation of autophagy and reduction of mutant protein synthesis (Supplementary Material, Fig. 6). These results might represent a new option for MJD and other polyQ diseases, as it could reach human clinics in a shorter frame than other therapeutic strategies tested until now.

\section{Materials and Methods}

\section{In vitro cordycepin treatment}

N2A cells and human neural cultures (derived from Induced pluripotent stem cells) were treated with $20 \mu \mathrm{M}$ and $200 \mu \mathrm{M}$ of cordycepin, respectively.

\section{Animals}

Adult C57/BL6 male mice (Charles River Laboratories, Barcelona, Spain) and 4-week-old transgenic MJD mice (29) were used in this experiment. Transgenic mice were breed in the animal house facility of the Centre for Neuroscience and Cell Biology of the University of Coimbra by backcrossing heterozygous MJD males with C57BL/ 6 females. All animals were housed in a temperature-controlled room maintained on a $12 \mathrm{~h}$ light-12 h dark cycle. Food and water were provided ad libitum. The experiments were carried out in accordance with the European Community directive $(86 / 609 / \mathrm{EEC})$ for the care and use of laboratory animals. The researchers received adequate training (FELASAcertified course) and certification to perform the experiments from the Portuguese authorities (Direcção Geral de Alimentação e Veterinária).

\section{Lentiviral vectors}

LV encoding for human full-length mutant Atx3Q72 and human wild-type Atx3 (Atx3-27Q) (2) were produced and quantified in HEK 293T cells using a four-plasmid system described previously (24).

\section{Neuroblastoma cells culture, infection and cordycepin treatment}

N2a cells obtained from the American Type Culture Collection cell biology bank (CCL-131) were maintained in Dulbecco's modified Eagle's medium (DMEM) supplemented with 10\% fetal bovine serum (FBS), $100 \mathrm{U} / \mathrm{ml}$ penicillin and $100 \mathrm{mg} / \mathrm{ml}$ streptomycin (Gibco) at $37^{\circ} \mathrm{C}$ in $5 \% \mathrm{CO}_{2} /$ air atmosphere. Cells were plated for infection with lentiviral particles expressing human full-length mutant Atx3Q72 or human wild-type Atx327Q. The infection was performed with $400 \mu \mathrm{l}$ of new medium, $8 \mu \mathrm{g} / \mathrm{ml}$ of hexadimethrin bromid (Sigma-Aldrich, St. Loius, Missouri, USA) and the LV in a total concentration of $10 \mathrm{ng}$ of p24 antigen per 100000 cells. The cells were then incubated at $37^{\circ} \mathrm{C}$ in $5 \% \mathrm{CO} 2 /$ air atmosphere and $8 \mathrm{~h}$ post-infection it was added $600 \mu \mathrm{l}$ of fresh medium to the cells. Transduced cells were stored at $-80^{\circ} \mathrm{C}$ in $10 \%$ Dimethyl sulfoxide $/ 90 \% \mathrm{FBS}$ for posterior use. For cordycepin ( $3^{\prime}$-deoxyadenosine, $100 \mathrm{mg} / \mathrm{ml}$ in DMSO, Sigma) treatment, cells were plated and $24 \mathrm{~h}$ after were treated with $20 \mu \mathrm{M}$ of cordycepin. After $48 \mathrm{~h}$ of treatment, cells were harvested for subsequent western blot analysis.

\section{Translation rate assay}

For the assessment of global protein synthesis, we used a previously described assay based on the incorporation of puromycin into the polypeptide chain during the translation process (25) and following the same procedure we used before (17).

\section{Autophagic flux measurement}

The autophagic flux was measured through the LC3B turnover assay, which is used to quantify the amount of LC3B-II that is 
delivered to the lysosomes (3). For that purpose, the levels of LC3B-II were measured in the presence and absence of ChQ (ChQ, Sigma and $100 \mu \mathrm{m}$ ), an inhibitor of autolysosomal degradation. The autophagic flux was calculated by subtracting the densitometric value of LC3B-II in samples without ChQ (LC3BII - ChQ) from the corresponding sample treated with ChQ (LC3B-II + ChQ) for each condition.

\section{Neural stem cell culture and differentiation}

Human neural stem cells (NSCs) derived from iPSCs from MJD fibroblasts were grown attached to flasks coated with Matrigel (BD Biosciences, Becton, Dickinson and Company, Franklin Lakes, New Jersey, USA) as previously described (4). Neural cultures were obtained by seeding $350000 \mathrm{NSCs} /$ well in $12 \mathrm{MW}$ plates with coverslips previously coated with poly-L-lysine and $1 \mu \mathrm{g} / \mathrm{ml}$ laminin (Sigma) and cultured with differentiation medium: DMEM-F12 supplemented with $2 \mu \mathrm{M}$ retinoic acid, $5 \mu \mathrm{M}$ forskolin, 2\% B27 and 1\% penicillin-streptomycin (Gibco, Thermo Fisher Scientific, Waltham, Massachusetts, USA). Cordycepin was administered at day 5 of differentiation $(200 \mu \mathrm{M})$, while the same volume of DMSO (solvent of cordycepin) was used as a control. The cells were fixed $48 \mathrm{~h}$ after adding the cordycepin/DMSO and were further processed for immunocytochemistry.

\section{Cell proliferation/viability}

The resazurin reduction assay is an accepted method to access cell viability (21). Briefly, $48 \mathrm{~h}$ after cordycepin administration, NSC cultures were incubated in differentiation medium containing $10 \%$ resazurin, at $37^{\circ} \mathrm{C}$ for $3 \mathrm{~h}$. Then, the absorbance of the medium was measured at $570 \mathrm{~nm}$ and $600 \mathrm{~nm}$. The cell viability was calculated as a percentage of the control cells (incubated with DMSO) by applying the following formula: cell viability $(\%)=$ [absorbance $(570-600 \mathrm{~nm})$ of treated cells $\times 100] /$ [absorbance $(570-600 \mathrm{~nm})$ of control cells].

\section{Lentiviral mouse model}

For the in vivo injection of LV, concentrated viral stocks encoding for mutant Atx3 (Atx3Q72) were thawed on ice and resuspended. Eight-week-old C57/BL6 male mice (Charles River Laboratories) were anesthetized by administration of a mixture of ketamine (100 mg/kg, Clorketam 1000, Vétaquinol) with xylazine $(10 \mathrm{mg} / \mathrm{kg}$, Rompun $^{\circledR}$, Bayer) by IP and stereotaxically injected into the right hemisphere of the striatum in the following coordinates: anteroposterior, $+0.6 \mathrm{~mm}$; lateral, $-1.8 \mathrm{~mm}$; ventral, $-3.3 \mathrm{~mm}$; mouth bar, 0 . Viral vectors content was determined by p 24 antigen ELISA (RETROtek, Gentaur, France) and injected at a rate of $0.25 \mu \mathrm{l} / \mathrm{min}$ (a single $1 \mu$ injection of $400000 \mathrm{ng}$ ) by means of an automatic injector (Stoelting Co., Wood Dale, IL, USA) into the mouse brain area through a 34-gauge blunt-tip needle linked to a Hamilton syringe (Hamilton, Reno, NV, USA). After injection, the syringe needle was left in place for an additional $5 \mathrm{~min}$ to allow the viral vector diffusion and to minimize backflow. One week poststereotaxic injection, the cordycepin treatment began. Treated animals received an IP $40 \mu \mathrm{l}$ injection of $20 \mathrm{mg} / \mathrm{Kg}$ of cordycepin (100 mg/mL in DMSO-NaCl 0.1\%), whereas a $40 \mu \mathrm{l}$ injection of the vehicle ( $\mathrm{NaCl} 0.1 \%)$ was administrated to the control group during 1 week or 4 consecutive weeks.

\section{Transgenic mouse model}

A transgenic mouse model for MJD (C57BL/6 background) expressing the N-terminal-truncated human Atx3 with 69 glutamine repeats together with an $\mathrm{N}$-terminal HA epitope in cerebellar Purkinje cell, driven by the L7 promoter was used (29). Animals were divided in two groups: the treated group, which was administered a daily IP $40 \mu$ injection of growing doses of cordycepin- $\mathrm{NaCl} 0.1 \%(15 \mathrm{mg} / \mathrm{Kg}$ in the first week, $20 \mathrm{mg} / \mathrm{Kg}$ in the second week and then $25 \mathrm{mg} / \mathrm{Kg}$ until the end of experiment), and the control group, which was injected with $40 \mu \mathrm{l}$ of the vehicle solution $(\mathrm{NaCl} 0.1 \%$ ). The treatment was maintained for 6 weeks and both groups included female and male mice in the same proportion.

\section{Behavioral assessment}

Transgenic mice groups were subjected to different motor tests (rotarod and footprint pattern analysis), every 2 weeks from the first treatment until the end of the experiment. An experienced operator performed the tests blindly, following the same procedure described before (20). All tests were performed in the same room after $1 \mathrm{~h}$ of acclimatization.

\section{Tissue preparation}

For the immunohistochemical procedures, animals received a sodium pentobarbital overdose, followed by a transcardial perfusion with $0.1 \mathrm{M}$ phosphate buffer solution and a $4 \%$ paraformaldehyde fixative solution (Fluka, Sigma, SigmaAldrich, St. Louis, Missouri, USA). The brains were then removed, post-fixed in $4 \%$ paraformaldehyde for $24 \mathrm{~h}$ and cryoprotected by incubation in $25 \%$ sucrose/phosphate buffer for $48 \mathrm{~h}$. Subsequently, brains were frozen and sectioned using a cryostat (LEICA CM3050 S). For the lentiviral mouse model, coronal sections of $20 \mu \mathrm{m}$ were obtained, while for the transgenic mice, $35 \mu \mathrm{m}$ sagittal brain sections were made. After sectioning, brain sections were collected and stored in 48-well trays, freefloating in $0.1 \mathrm{M}$ phosphate buffer solution supplemented with $0.05 \mu \mathrm{M}$ sodium azide and stored at $4^{\circ} \mathrm{C}$ until further usage. For western blot analysis, animals were sacrificed through sodium pentobarbital overdose without transcardial perfusion. Animals' blood was collected and sent to a clinical and pathological analyses laboratory for analyses of the hepatic enzymes: ALT and ALP. Brains were removed and dissected fresh for collection of striatal punches using a Harris Uni-Core pen, with $2.0 \mathrm{~mm}$ diameter (Ted Pella Inc., Redding, California, USA). Brain punches samples were then stored at $-80^{\circ} \mathrm{C}$ until posterior processing.

\section{Western blot}

For protein extraction, brain tissue or cells were first lysed in RIPA-buffer solution (50 mm Tris $\mathrm{HCl} \mathrm{pH}=8 ; 150 \mathrm{~nm} \mathrm{NaCl} ; 1 \%$ NP-40; $0.5 \%$ sodium deoxycholate; $0.1 \%$ SDS) containing protease inhibitors (Roche diagnostics, Risch-Rotkreuz, Switzerland), followed by sonication of $4 \mathrm{~s}$ ultrasound chase (1 pulse/s). Total protein lysates were stored at $-80^{\circ} \mathrm{C}$ and protein concentration was determined through the Pierce ${ }^{\mathrm{TM}}$ BCA Protein Assay Kit (Thermo Scientific, Thermo Fisher Scientific, Waltham, Massachusetts, USA). Sixty micrograms of protein extract were resolved in SDS-polyacrylamide gels $(10 \%)$. Then proteins were transferred onto polyvinylidene difluoride membrane (GE Healthcare) according to standard protocols. Immunoblotting was performed using the following antibodies: mouse monoclonal anti-Atx3 (Millipore, 1:1000), mouse anti-tubulin (Sigma; 1:5000), anti-AMPK (1:1000, Cell Signaling), anti-phosphoAMPK (1:2000, Cell Signaling), rabbit anti-mTOR (1:1000; Cell Signaling), rabbit anti-P-mTOR (Ser2448) (1:1000; Cell Signaling), 
rabbit anti-ULK1 (1:1000; Cell Signaling), rabbit anti-phosphoULK1 (Ser555) (1:1000; Cell Signaling), rabbit anti-LC3B (1:1000, Cell Signaling), rabbit anti-SQSTM1 (1:1000, Cell Signaling), mouse anti-puromycin (Millipore, 1:20000) and mouse antiGAPDH (1:3000; Abcam). Semi-quantitative analysis was carried out based on the optical density of the same gel (Quantity One ${ }^{\circledR} 1$ D image analysis software version 4.4; Bio-Rad).

\section{Immunochemical procedures}

Immunocytochemistry. Immunocytochemistry was performed based on protocols described previously $(13,17)$. Briefly, cells were washed with Phosphate-buffered saline and fixed with $4 \%$ paraformaldehyde (Sigma) at room temperature for $15 \mathrm{~min}$. The cells were then permeabilized with $1 \%$ Triton $^{\mathrm{TM}} \mathrm{X}-100$ (Sigma) followed by $1 \mathrm{~h}$ of blocking with 3\% Bovine Serum Albumin (Sigma) in PBS. The primary antibodies were incubated with cells overnight at $4^{\circ} \mathrm{C}$. Cells were then washed with PBS following incubation with the secondary antibodies for $2 \mathrm{~h}$ at room temperature. Subsequently, cells were washed with PBS and nuclei were stained with $4^{\prime}, 6^{\prime}$-Diamidino-2'-phenylindole (1:5000, Applichem) for $5 \mathrm{~min}$ at room temperature. After a washing step, the coverslips were mounted on microscope slides using Mowiol as a mounting medium. Images were acquired with $20 \times$ objective in a Zeiss Axio Imager Z2.

Immunohistochemistry. Immunocytochemistry assay was performed as previously described $(18,19)$. Briefly, the immunohistochemistry for light microscopy began by incubating free floating the brain slices in phosphate buffer solution containing $0.1 \%$ diphenylhydrazine for $1 \mathrm{~h}$ at $37^{\circ} \mathrm{C}$. Sections were then incubated in blocking solution $[0.1 \%$ TritonX 100 containing 10\% Normal Goat Serum (NGS, Gibco) in phosphate buffer solution], followed by overnight incubation at $4^{\circ} \mathrm{C}$ with respective primary antibodies. Posteriorly, sections were incubated with secondary antibody for $2 \mathrm{~h}$ at room temperature. The secondary antibody (1:200) used was biotinylated and followed a reaction with the Vectastain elite avidin-biotin-peroxidase kit and by 3,3'-diaminobenzidine substrate (both from Vector Laboratories). Sections were then mounted over microscope slides, hydrated with $\mathrm{H}_{2} \mathrm{O}$ and then dehydrated by passing through an increased degree of ethanol solutions (ETOH 75, 96 and 100\%) and finally xylene. Slides were covered with mounting medium Eukitt (O. Kindler $\mathrm{GmbH}$ \& $\mathrm{CO}$, Freiburg, Germany) and coverslipped. In the fluorescencelabeling protocol, sections were incubated in a $0.3 \%$ Triton $10 \%$ NGS 0.1 phosphate buffer blocking solution for $1 \mathrm{~h}$ at room temperature and then overnight at $4{ }^{\circ} \mathrm{C}$ with the appropriate primary antibodies. Afterwards, slices were incubated in the secondary antibody for $2 \mathrm{~h}$ at room temperature. Sections were then incubated with DAPI solution (1:5000) for $10 \mathrm{~min}$ at room temperature, washed and mounted on Mowiol mounting medium.

Immunochemical antibodies. Samples were incubated with the following primary antibodies: mouse anti- $\beta$ III tubulin (1:500, Invitrogen, Thermo Fisher Scientific, Waltham, Massachusetts, USA), rabbit anti-ubiquitin (1:300, Enzo Life Sciences, Farmingdale, New York, USA), anti-ubiquitin (1:500; Merck Millipore, Burlington, Massachusetts, USA), anti-NeuN (1:1000, Chemicon International, Thermo Fisher Scientific, Waltham, Massachusetts, USA), anti-HA (HA tag, 1:1000, InvivoGen, San Diego, California, USA) and anti-Calbindin (clone D-28 K, 1:1000, Chemicon).

\section{Cresyl violet staining}

Brain slices were mounted over microscope slides, stained with cresyl violet dye and dehydrated by passing through an increased degree of ethanol solutions (ETOH 75, 96 and 100\%) and finally xylene. Slides were then covered with mounting medium Eukitt (O. Kindler GmbH \& CO, Freiburg, Germany) and cover slipped.

\section{Immunochemistry quantitative analysis}

Evaluation of the NeuN depleted volume. The extent of neuronal lesion in the striatum was photographed, with a $20 \times$ objective, eight sections per animal $(20 \mu \mathrm{m}$ thickness sections at $200 \mu \mathrm{m}$ intervals) stained with the neuronal marker NeuN, thus obtaining a complete rostrocaudal sampling of the striatum. A semi-automated image analysis software package was used to measure the area of the neuronal lesion (Image J software, USA). The final depleted volume was then estimated with the following formula: volume $=d^{*}(a 1+a 2+a 3)$, where $d$ is the distance between serial sections $(200 \mu \mathrm{m})$ and $\mathrm{a} 1+\mathrm{a} 2+\mathrm{a} 3$ are depleted areas for individual serial sections. Data are represented as mean value of the final NeuN depleted volume of the eight sections relative to the striatum total volume.

Quantification of ubiquitin inclusions number and size. Coronal sections for a complete rostrocaudal sampling (eight sections) of the striatum were scanned with a $20 \times$ objective using a Zeiss Axiovert $200 \mathrm{M}$ imaging microscope Zeiss (Zeiss, Germany), thus including regions with ubiquitin-positive aggregates. For each animal, all inclusions were manually counted using a semiautomated image-analysis software package (Image J software, USA). The size of the aggregates was automatically counted using the CellProfiler software.

Quantification of mutant Atx3 aggregates and Purkinje cells.. Fluorescence images of 8 sagittal sections, spanning $280 \mu \mathrm{m}$ between them, of the entire cerebellum, stained with anti$\mathrm{HA}$, anti-Calbindin and DAPI were acquired with a Zeiss Axio Imager $\mathrm{Z} 2$ microscope using a $20 \times$ objective. For each section, the number of cells with mutant Atx3 aggregates and Purkinje cells were manually counted in all cerebellar lobules using an image analysis software (ZEN 2.1 lite, Zeiss). Data are represented as mean value of the number of cells with aggregates of the eight sections analyzed or the number of Purkinje cells normalized with the number of aggregates per section.

Quantification of cerebellar lobular volume. Fluorescence images of eight sagittal sections, spanning $280 \mu \mathrm{m}$ between them, of the entire cerebellum, stained with cresyl violet were acquired with a Zeiss Axio Imager Z2 microscope using a 20x objective. For each section, the area of each cerebellar lobe was measured using an image analysis software (ZEN 2.1 lite, Zeiss). The final lobe volume was then estimated with the following formula: volume $=d^{*}(a 1+a 2+a 3)$, where $d$ is the distance between serial sections $(280 \mu \mathrm{m})$ and $\mathrm{a} 1+\mathrm{a} 2+\mathrm{a} 3$ are lobe areas for individual serial sections. Data are represented as mean value of the cerebellar lobe volume of the eight sections analyzed normalized with cerebellum total volume.

Statistical analysis. Statistical analysis was performed using Student's t-test or ANOVA for multiple comparisons using GraphPad software (La Jolla, USA). Values of $P<0.05$ were considered statistically significant. 


\section{Supplementary Material}

Supplementary Material is available at HMG online.

\section{Acknowledgements}

We acknowledge the Light Microscopy Unit of CBMR-UAlg, which is partially supported by national Portuguese funding FCT: UID/BIM/04773/2013 CBMR and PPBI-POCI-01-0145-FEDER022122. We thank Dr Célia Aveleira for the valuable scientific review of the manuscript.

Conflict of Interest statement. None declared.

\section{Funding}

European Union through the European social fund, funds Fundo Europeu de Desenvolvimento Regional through the Competitive Factors Operational Program-COMPETE, POPH and QREN; the French Muscular Dystrophy Association (AFM-Téléthon) (project \#18776); Ataxia UK; Fundação para a Ciência e Tecnologia (PhD fellowship SFRH/BD/133192/2017 to A.M.). National funds to FCT - Fundação para a Ciência e a Tecnologia, I.P., project reference UID/BIM/04773/2013 CBMR.

\section{References}

1. Alves, S., Nascimento-Ferreira, I., Auregan, G., Hassig, R., Dufour, N., Brouillet, E., Pedroso de Lima, M.C., Hantraye, P., Pereira de Almeida, L. and Déglon, N. (2008a) Allele-specific RNA silencing of mutant ataxin-3 mediates neuroprotection in a rat model of Machado-Joseph disease. PLoS One, 3, e3341.

2. Alves, S., Régulier, E., Nascimento-Ferreira, I., Hassig, R., Dufour, N., Koeppen, A., Carvalho, A.L., Simões, S., de Lima, M.C.P., Brouillet, E. et al. (2008b) Striatal and nigral pathology in a lentiviral rat model of Machado-Joseph disease. Hum. Mol. Genet., 17, 2071-2083.

3. Aveleira, C.A., Botelho, M., Carmo-Silva, S., Pascoal, J.F., Ferreira-Marques, M., Nóbrega, C., Cortes, L., Valero, J., SousaFerreira, L., Álvaro, A.R. et al. (2015) Neuropeptide Y stimulates autophagy in hypothalamic neurons. Proc. Natl. Acad. Sci. U. S. A., 112, E1642-E1651.

4. Carmona, V., Cunha-Santos, J., Onofre, I., Simões, A.T., Vijayakumar, U., Davidson, B.L. and Pereira de Almeida, L. (2017) Unravelling endogenous microRNA system dysfunction as a new pathophysiological mechanism in MachadoJoseph disease. Mol. Ther., 25, 1038-1055.

5. Cha, J.-Y., Ahn, H.-Y., Cho, Y.-S. and Je, J.-Y. (2013) Protective effect of cordycepin-enriched Cordyceps militaris on alcoholic hepatotoxicity in Sprague-Dawley rats. Food Chem. Toxicol., 60, 52-57.

6. Cortes, C.J. and La Spada, A.R. (2015) Autophagy in polyglutamine disease: imposing order on disorder or contributing to the chaos? Mol. Cell. Neurosci., 66, 53-61.

7. Cummings, C.J. and Zoghbi, H.Y. (2000) Trinucleotide repeats: mechanisms and pathophysiology. Annu. Rev. Genomics Hum. Genet., 1, 281-328.

8. Cunha-Santos, J., Duarte-Neves, J., Carmona, V., Guarente, L., Pereira de Almeida, L. and Cavadas, C. (2016) Caloric restriction blocks neuropathology and motor deficits in Machado-
Joseph disease mouse models through SIRT1 pathway. Nat. Commun., 7, 11445.

9. Doss-Pepe, E.W., Stenroos, E.S., Johnson, W.G. and Madura, K. (2003) Ataxin-3 interactions with rad23 and valosincontaining protein and its associations with ubiquitin chains and the proteasome are consistent with a role in ubiquitin-mediated proteolysis. Mol. Cell. Biol., 23, 6469-6483.

10. Hewitt, M., Enoch, S.J., Madden, J.C., Przybylak, K.R. and Cronin, M.T.D. (2013) Hepatotoxicity: a scheme for generating chemical categories for read-across, structural alerts and insights into mechanism(s) of action. Crit. Rev. Toxicol., 43, 537-558.

11. Kim, J., Kundu, M., Viollet, B. and Guan, K.-L. (2011) AMPK and mTOR regulate autophagy through direct phosphorylation of ULK1. Nat. Cell Biol., 13, 132-141.

12. Maciel, P., Costa, M.C., Ferro, A., Rousseau, M., Santos, C.S., Gaspar, C., Barros, J., Rouleau, G.A., Coutinho, P. and Sequeiros, J. (2001) Improvement in the molecular diagnosis of Machado-Joseph disease. Arch. Neurol., 58, 1821-1827.

13. Mendonça, L.S., Nóbrega, C., Hirai, H., Kaspar, B.K. and Pereira de Almeida, L. (2015) Transplantation of cerebellar neural stem cells improves motor coordination and neuropathology in Machado-Joseph disease mice. Brain, 138, 320-335.

14. Moulis, M. and Vindis, C. (2017) Methods for measuring autophagy in mice. Cell, 6, 14.

15. Nascimento-Ferreira, I., Nóbrega, C., Vasconcelos-Ferreira, A., Onofre, I., Albuquerque, D., Aveleira, C., Hirai, H., Déglon, N. and Pereira de Almeida, L. (2013) Beclin 1 mitigates motor and neuropathological deficits in genetic mouse models of Machado-Joseph disease. Brain, 136, 2173-2188.

16. Nascimento-Ferreira, I., Santos-Ferreira, T., Sousa-Ferreira, L., Auregan, G., Onofre, I., Alves, S., Dufour, N., Colomer Gould, V.F., Koeppen, A., Déglon, N. and Pereira de Almeida, L. (2011) Overexpression of the autophagic beclin-1 protein clears mutant ataxin-3 and alleviates Machado-Joseph disease. Brain, 134, 1400-1415.

17. Nóbrega, C., Carmo-Silva, S., Albuquerque, D., VasconcelosFerreira, A., Vijayakumar, U.-G., Mendonça, L., Hirai, H. and Pereira de Almeida, L. (2015) Re-establishing ataxin-2 downregulates translation of mutant ataxin-3 and alleviates Machado-Joseph disease. Brain, 138, 3537-3554.

18. Nóbrega, C., Nascimento-Ferreira, I., Onofre, I., Albuquerque, D., Conceição, M., Déglon, N. and de Almeida, L.P. (2013a) Overexpression of mutant ataxin-3 in mouse cerebellum induces ataxia and cerebellar neuropathology. Cerebellum, 12, 441-455.

19. Nóbrega, C., Nascimento-Ferreira, I., Onofre, I., Albuquerque, D., Déglon, N. and Pereira de Almeida, L. (2014) RNA interference mitigates motor and neuropathological deficits in a cerebellar mouse model of Machado-Joseph disease. PLoS One, 9, e100086.

20. Nóbrega, C., Nascimento-Ferreira, I., Onofre, I., Albuquerque, D., Hirai, H., Déglon, N. and de Almeida, L.P. (2013b) Silencing mutant ataxin-3 rescues motor deficits and neuropathology in Machado-Joseph disease transgenic mice. PLoS One, 8, e52396.

21. O'Brien, J., Wilson, I., Orton, T. and Pognan, F. (2000) Investigation of the Alamar Blue (resazurin) fluorescent dye for the assessment of mammalian cell cytotoxicity. Eur. J. Biochem., 267, 5421-5426.

22. Paterson, R.R.M. (2008) Cordyceps: a traditional Chinese medicine and another fungal therapeutic biofactory? Phytochemistry, 69, 1469-1495. 
23. Paulson, H.L., Das, S.S., Crino, P.B., Perez, M.K., Patel, S.C., Gotsdiner, D., Fischbeck, K.H. and Pittman, R.N. (1997) Machado-Joseph disease gene product is a cytoplasmic protein widely expressed in brain. Ann. Neurol., 41, 453-462.

24. Pereira de Almeida, L., Zala, D., Aebischer, P. and Déglon, N. (2001) Neuroprotective effect of a CNTF-expressing lentiviral vector in the quinolinic acid rat model of Huntington's disease. Neurobiol. Dis., 8, 433-446.

25. Schmidt, E.K., Clavarino, G., Ceppi, M. and Pierre, P. (2009) SUnSET, a nonradioactive method to monitor protein synthesis. Nat. Methods, 6, 275-277.

26. Simões, A.T., Gonçalves, N., Nobre, R.J., Duarte, C.B. and Pereira de Almeida, L. (2014) Calpain inhibition reduces ataxin-3 cleavage alleviating neuropathology and motor impairments in mouse models of Machado-Joseph disease. Hum. Mol. Genet., 23, 4932-4944.

27. Sudarsky, L. and Coutinho, P. (1995) Machado-Joseph disease. Clin. Neurosci., 3, 17-22.
28. Taroni, F. and DiDonato, S. (2004) Pathways to motor incoordination: the inherited ataxias. Nat. Rev. Neurosci., 5, 641-655.

29. Torashima, T., Koyama, C., Iizuka, A., Mitsumura, K., Takayama, K., Yanagi, S., Oue, M., Yamaguchi, H. and Hirai, H. (2008) Lentivector-mediated rescue from cerebellar ataxia in a mouse model of spinocerebellar ataxia. EMBO Rep., 9, 393-399.

30. Wong, P.-M., Puente, C., Ganley, I.G. and Jiang, X. (2013) The ULK1 complex. Autophagy, 9, 124-137.

31. Wong, Y.Y., Moon, A., Duffin, R., Barthet-Barateig, A., Meijer, H.A., Clemens, M.J. and de Moor, C.H. (2010) Cordycepin inhibits protein synthesis and cell adhesion through effects on signal transduction. J. Biol. Chem, 285, 2610-2621.

32. Wu, Y., Peng, Y. and Wang, Y. (2015) An insight into advances in the pathogenesis and therapeutic strategies of spinocerebellar ataxia type 3. Rev. Neurosci., 26, 95-104. 